Rahel Beyer

\title{
Sprachkontaktinduzierte Variation in den öffentlichen Bekanntmachungen der Stadt Luxemburg im 19. Jahrhundert
}

\section{Einleitung}

„Die beiden Sprachräume grenzen also seit ältester Zeit aneinander und haben sich gerade in Auseinandersetzung miteinander entwickelt“ (Zollna 2004: 3192). Diese Aussage, den Sprachkontakt zwischen Deutsch und Französisch betreffend, lässt sich v.a. auf der Basis von Untersuchungen zu Elsass-Lothringen, Belgien und der Schweiz treffen. Die dort zu findenden Sprachkontaktphänomene betreffen sowohl die jeweilige Varietät des Französischen als auch des Deutschen sowie jegliche Ebenen des Sprachsystems. Sie wurden in vielen Arbeiten analysiert und beschrieben (für einen Überblick vgl. Zollna 2004).

Daneben findet sich auch in Luxemburg ein Kontaktraum zwischen Germania und Romania, der indes bisher weniger Beachtung gefunden hat. Dies gilt insbesondere für sprachstrukturelle Aspekte. Bestehende Forschung in Bezug auf Deutsch in Luxemburg konzentriert sich typischerweise auf makrosoziolinguistische Aspekte des Sprachgebrauchs und Spracheinstellungen (vgl. Fehlen 2009; Fröhlich 1989; Horner \& Weber 2008; Sieburg 2013). Daneben beschäftigen sich nur wenige Arbeiten mit sprachlichen Phänomenen oder fokussieren gar gezielt Erscheinungen des Sprachkontakts. Meistens bleibt es bei kursorischen Randnotizen und einer losen Sammlung von Beispielen, die sich zudem auf die Lexik konzentrieren (vgl. Fröhlich \& Hoffmann 1997; Sieburg 2012). Ein paar detailliertere Studien nehmen die Zeit vom Ende des 14. bis zum Ende des 17. Jahrhunderts (vgl. Ravida 2012; Solms \& Wegera 1999) oder das 20. Jahrhundert (Magenau 1964) in den Blick. Ein umfassendes Bild der Entwicklung des Deutschen und seiner Grammatik in Luxemburg unter systematischer Einbeziehung des Sprachkontaktes v.a. mit dem Französischen kann unterdessen bislang nicht beschrieben werden, zumal für das gesamte 18. und 19. Jahrhundert eine vollständige Forschungslücke zu konstatieren ist. 
Charakteristikum Luxemburgs ist seine weit zurückreichende Mehrsprachigkeit und seine wechselvolle politische Geschichte. ${ }^{1}$ Für das 19. Jahrhundert lassen sich v.a. die französische Herrschaft von 1795-1814, die Gründung des Großherzogtums Luxemburgs 1815 (allerdings als persönlicher Besitz von Wilhelm I., König der Niederlande) und die Abtretung eines großen Teils des Staatsterritoriums im Zusammenhang mit der Belgischen Revolution im Jahr 1839 nennen. Sprachgeographisch liegt bzw. lag Luxemburg auf der germanisch-romanischen Sprachgrenze, was eine lange Geschichte der Mehrsprachigkeit und des Sprachkontakts mit sich bringt. So gab es im 19. Jahrhundert zwei Amtssprachen: Französisch wurde im „quartier wallon“ gebraucht, das jedoch später Belgien einverleibt wurde und sich heute westlich der luxemburgischen Staatsgrenze befindet. Östlich schloss sich das „quartier allemand“ an, das weitgehend dem heutigen Territorium des Großherzogtums entspricht. Jedoch auch nach der Teilung im Jahre 1839, seit der Luxemburg vollständig und einzig dem germanophonen Gebiet angehört, bleibt die Mehrsprachigkeit erhalten (Fröhlich 1989).

Angesichts dieses engen Sprachkontakts ist davon auszugehen, dass Spuren des Französischen im Deutschen zu finden sind und sich auch als Einflussgröße im Wandel der Sprache erkennen lassen. Ziel des vorliegenden Beitrags ist es dementsprechend, den Wandel des Deutschen in Luxemburg im 19. Jahrhundert zu beleuchten und in diesem Zusammenhang insbesondere Aspekte des Sprachkontakts mit dem Französischen zu berücksichtigen. Dazu werden exemplarisch drei Phänomene betrachtet, ihre Entwicklung im Laufe des Jahrhunderts analysiert und die Beobachtungen im Hinblick sowohl auf bekannte Entwicklungstendenzen des Deutschen als auch auf Transferenzen aus dem Französischen eingeordnet. Um zu überprüfen, inwiefern sich sprachliche Strukturen mit kontaktsprachlichem Einfluss anders verhalten als sprachinterne Entwicklungen, handelt es sich bei einem der drei Phänomene um eine Variable ohne Rückbindung an einen französischen Kontext.

Der Beitrag gliedert sich in vier Teile: Zunächst wird das Projekt „Sprachstandardisierung unter Mehrsprachigkeitsbedingungen: Deutsch in Luxemburg (1795-1920)“ erläutert, das den Kontext der nachfolgenden Analysen darstellt (Abschnitt 2). In Abschnitt 3 wird das Korpus für die Analysen hinsichtlich Charakteristika, Größe und Aufbereitung beschrieben. Die Analyse und Interpretation der drei Phänomene (Assimilation von Fremdwörtern aus dem Französischen, Adverbialisierung der Tageszeitangaben und Genusdifferenzierung beim Zahlwort ,zwei') findet sich in Abschnitt 4. Abschnitt 5 fasst die wesentlichen Erkenntnisse zusammen.

1 Für eine ausführlichere Darstellung der luxemburgischen Geschichte vgl. Pauly (2011). 


\section{Zum Projekt „Sprachstandardisierung unter Mehrsprachigkeitsbedingungen: Deutsch in Luxemburg (1795-1920)“}

Die in diesem Beitrag dargelegte Forschung wurde im Rahmen des binationalen Projekts „Sprachstandardisierung unter Mehrsprachigkeitsbedingungen: Deutsch in Luxemburg (1795-1920)“ durchgeführt. Ausgangspunkt ist die grundlegende Annahme, dass Sprachkontakt und Mehrsprachigkeit in Bezug auf Sprachwandel eine relevante Einflussgröße darstellen (Heine \& Kuteva 2005). Nichtsdestotrotz ist ihr Einfluss im Zusammenhang mit Sprachstandardisierung weitestgehend unerforscht. Um diese Lücke zu füllen, widmet sich das Projekt der Standardisierung des Deutschen in Luxemburg, wo seit langem sowohl verschiedene germanische Varietäten untereinander (Deutsch / Moselfränkisch / emergierendes Luxemburgisch, kolloquiales Deutsch) als auch Deutsch mit Französisch in Kontakt stehen.

Das Projekt ist empirisch ausgerichtet und stützt sich auf ein umfangreiches Korpus einer in sprachgeschichtlichen Studien bis dato unberücksichtigten Textsorte, nämlich zweisprachigen öffentlichen Bekanntmachungen der Stadt Luxemburg aus dem 19. Jahrhundert. Der Bereich der Verwaltung bietet insofern ein hervorragendes Umfeld für das Forschungsziel, als er einen sozial bedeutenden Sprachgebrauchstyp konstituiert. Um systemische wie funktionale Aspekte gleichermaßen zu berücksichtigen, umfasst die Rekonstruktion des Standardisierungsprozesses des Deutschen strukturelle Prozesse wie die Variation und Transferenz sprachlicher Merkmale als auch relevante SprachenmanagementAktivitäten (z.B. Verordnungen, Gesetze), metasprachliche Kommentare in den Bekanntmachungen und Spracheinstellungsäußerungen in Zeitungen. Auf diese Weise ergibt sich ein detailliertes Bild davon, wie Deutsch in die Domäne der Verwaltung Einzug gehalten hat (= Statusstandardisierung), wie es sich strukturell über die Zeit hinweg entwickelt hat (= Korpusstandardisierung) und wie technische und lexikalische Professionalisierung der öffentlichen Bekanntmachungen im Untersuchungszeitraum fortgeschritten sind (= funktionale Elaboration).

\section{Korpus}

Die Datengrundlage für den hier vorgestellten Teil der Untersuchung bildet ein Korpus von 2.348 öffentlichen Bekanntmachungen ${ }^{2}$, deren Aushang auf dem

2 Wir danken der Leiterin des Luxemburger Stadtarchivs, Dr. Evamarie Bange, für die freundliche Genehmigung der Analyse der Dokumente.. 
Gebiet des späteren Großherzogtums seit dem 18. Jahrhundert gängige Praxis war, um einem sich etablierenden Bürgertum Informationen und Entscheidungen zu Verwaltungsangelegenheiten zugänglich zu machen. Typische Orte für den Aushang der teils sehr großformatigen Plakate waren strategische und viel frequentierte Orte wie Rathäuser oder Kirchen. Auf diese Weise trugen die Bekanntmachungen zur Nutzung des öffentlichen Raums für Kommunikation und Entstehung eines öffentlichen Sprachraums bei. Thematisch wird ein weites Spektrum an administrativen Belangen behandelt wie etwa Holzverkauf, Verhaltensregeln zur Karnevalszeit, Besteuerung von Hunden u.v.m. Die Textgröße der einzelnen Anschlagzettel variiert zwischen 700 und 51.000 Zeichen, so dass sich in der Summe eine Zahl von 1,8 Millionen Wortformen ergibt, die für die Untersuchung genutzt werden können.

Wichtigstes Merkmal im Zusammenhang mit dem beschriebenen Erkenntnisinteresse ist die Edition ungefähr der Hälfte der Plakate als Paralleltext, d.h. als zwei äquivalente Texte selben Inhalts, einmal in Französisch und einmal in Deutsch (die übrigen sind einsprachig deutsch, französisch und selten niederländisch ${ }^{3}$ ). Somit handelt es sich um ein homogenes Korpus mit konstanten kontextuellen Bedingungen und derselben kommunikativen Funktion der beiden Sprachversionen, die jeweils eine Vergleichbarkeit der beiden Texte zulassen. Da zumindest für die Anfangszeit davon auszugehen ist, dass es sich bei den deutschen Texten um eine unmittelbare Übersetzung der französischen Originalversion handelt, ist umso mehr mit Transferenzen zu rechnen. Diese können durch die parallele Anordnung der beiden Versionen direkt aufgedeckt werden. Während die Kontrastive Linguistik sonst beispielsweise den Umweg über die Konsultation von Grammatikbeschreibungen und Wörterbücher zur Rekonstruktion eines Einflusses nehmen muss und immer nur indirekt auf Transferenzen schließen kann, können in den hier vorliegenden Paralleltexten Realisierungen in der einen Sprache unmittelbar auf Form- oder Wortgleichheit in der anderen Sprache überprüft werden und damit Replikation nachgewiesen werden. Dies ist für Untersuchungen im Umfeld der Sprachkontaktforschung umso attraktiver, als dass gerade auf der Ebene von grammatischen Phänomenen ein Nachweis von kontaktinduzierten Veränderungen immer wieder als problematisch diskutiert wird (vgl. Heine 2009; Milroy 1992). Außerdem wird auf diese Weise Forderungen nachgegangen, Paralleltextkorpora auch für historisch-diachrone Fragestellungen heranzuziehen (vgl. Claridge 2008).

3 Für die genauen Verhältnisse der Sprachenwahlen in den öffentlichen Bekanntmachungen vgl. Beyer et al. (2014). 
Da die Sammlung des Stadtarchivs Luxemburg einen Zeitraum von der französischen Annexion im Jahr 1795 bis zum Ende des ersten Weltkriegs abdeckt, ist auch die erforderliche zeitliche Tiefe gegeben, um eine diachrone Analyse durchzuführen. Es handelt sich bei dem Korpus somit um eines der wenigen historischen Paralleltextkorpora. ${ }^{4}$

Zur Erschließung des Korpus wurden zunächst aus insgesamt über 5.600 inventarisierten Bekanntmachungen anhand von soziolinguistisch-historischen Kriterien 2.348 Dokumente ausgewählt, mit einem Großscanner erfasst und in einem weiteren Schritt manuell text-digitalisiert. Alle Daten wurden in ein integriertes Datenbank-, Such- und Taggingtool (IDaSTO, vgl. Beyer 2015) eingestellt. Diese Aufbereitung macht es möglich, das Korpus maschinell zu durchsuchen, die Textstellen in beiden Sprachen parallel angezeigt zu bekommen und mit Tags zu versehen, die die einzelnen Realisierungen klassifizieren. Über die statistische Auswertung der vergebenen Tags kann eine quantitative Analyse von strukturellen Phänomenen vorgenommen werden, die Aufschluss über die Verteilung ihrer Varianten im Laufe des Untersuchungszeitraums liefert.

\section{Sprachkontakt und Standardisierung}

Da die sprachkontaktinduzierte Variation im Kontext der (auslaufenden) Standardisierung, d.h. einer besonderen Form von Sprachwandel (vgl. Mattheier 1998: 826) betrachtet wird, geht es nicht darum, punktuell Belege von Sprachkontakt zu finden und zu beschreiben, sondern ihren Entwicklungsverlauf nachzuverfolgen. Dabei stellt sich die Frage, wie sich das Aufeinandertreffen der beiden Phänomene gestaltet. Angesichts der zeitlichen Situierung in der Phase der abschließenden Bereinigung (nach Besch 2003 ab Mitte des 18. Jahrhunderts) ist grundsätzlich für die Entwicklungen auf der strukturellen Ebene mit Variantenabbau zu rechnen. Zusätzlicher Sprachkontakt kann hingegen die Quelle für Variation sein - resultierend aus Transferenzen und Replikation bzw. aus der Variantenwahl nach dem Modell der Kontaktsprache. Wenn sich Letzteres durchsetzt, gibt es quantitativ zwar ebenfalls Variantenreduktion, diese fällt jedoch qualitativ anders aus als in Gebieten ohne solch engen Sprachkontakt (vgl. Rash 2002). Unter Umständen könnte es aber auch zu anhaltender Variation, d.h. zu nebeneinander existierenden Formen für ein und dieselbe Struktur kommen. Oder aber Standardisierung und Normierung verfügen über solch eine Kraft, dass sie sich auch in Kontaktsituationen durchsetzen. In diesem Fall wäre es dann

4 Vgl. für eine ausführliche Korpusbeschreibung Gilles \& Ziegler (2013). 
möglich, dass der Prozess allerdings länger dauert, da es u.U. mehr Varianten gibt, die abgebaut werden müssen. Nicht zuletzt ist ein unterschiedlicher Niederschlag des Sprachkontakts in verschiedenen strukturellen Ebenen denkbar.

\subsection{Assimilation von Fremdwörtern aus dem Französischen}

Die erste Variable stammt aus dem Bereich der Schreibung und betrifft entlehnte Wörter. „Mit dem Entlehnungsprozess [...] beginnt gleichzeitig der Einbürgerungsprozess der übernommenen Einheiten, d.h. die formale und semantische Integration“ (Zastrow 2015: 1), die an dieser Stelle analysiert werden soll. Dabei sind im Kontext des französisch-deutschen Sprachkontakts Entlehnungen aus dem Französischen von zentralem Interesse. Aufgrund der dominanten Stellung des Französischen in Europa seit dem 12. Jahrhundert haben mehrere Tausend französische Wörter bzw. Wortteile ihren Weg in den deutschen Wortschatz gefunden (Baum 2000). Angesichts des noch intensiveren Sprachkontakts, wie er an Sprachgrenzen stattfindet, ist für Luxemburg aber davon auszugehen, dass dort verstärkt Gebrauch davon gemacht wird. In der Folge stellt sich die Frage, inwiefern sich diese Tatsache wiederum auf die Integration der Fremdwörter auswirkt.

Da es in der folgenden Analyse um die Überprüfung einer formalen „Anpassung an heimische Strukturgesetze“ gehen soll, wird im Weiteren mit Zastrow (2015: 49; wiederum Heller 1981: 34 folgend) der Begriff „Assimilation“ verwendet. Wenn es sich dann wie im vorliegenden Fall um schriftliche Daten handelt, ist von einer Angleichung zunächst der graphematische Bereich betroffen, der jedoch insofern an die phonologische Ebene gekoppelt ist, als ein Graphem als kleinste bedeutungsunterscheidende Einheit $\mathrm{zu}$ definieren ist, deren Bezeichnetes ein Phonem ist (Zastrow 2015: 28). Dementsprechend kann die Verschriftlichung eines Wortes Auskunft über dessen Aussprache liefern bzw. findet die Aussprache Niederschlag in der Schreibung. Diese Phonem-Graphem-Korrespondenzen sind sprachenspezifisch, über (geschriebene) Fremdwörter kommen jedoch auch fremde Phonem-Graphem-Beziehungen in eine Sprache. Im Zuge einer Assimilation können diese im Wesentlichen auf zwei verschiedenen Wegen angepasst werden. Erstens kann „eine Substitution der Fremdgrapheme wie in Frisör, Büro etc. [erfolgen], die das Ziel hat, die quellsprachige Aussprache weitgehend zu erhalten“ (Munske 1997: 98), dafür jedoch auf das heimische Grapheminventar zurückgreift. Alternativ kann eine Anpassung auf der phonologischen Ebene stattfinden, d.h. ,[d]ie quellsprachige Schreibung wird weitgehend erhalten, aber die Zeichenfolge wird nach dem Muster indigener Graphem-Phonem-Beziehungen ausgesprochen“ (Munske 1997: 99) (Leseaussprache). Grundsätzlich kann es aber auch vorkommen, dass Fremdwörter gar nicht assimiliert werden und ihre fremden 
Grapheme bzw. Phonem-Graphem-Beziehungen behalten. Gerade für Luxemburg mit seiner engen Kontaktsituation stellt sich die Frage, inwiefern hier Assimilation in gleichem Maße und auf gleiche Weise stattfindet. Vor dem Hintergrund einer Interpretation von Assimilation als Weg, Fremdwörter ,,jedem Sprecher/Schreiber - ungeachtet seiner fremdsprachigen Ausbildung - verfügbar zu machen“ (Munske 1997: 89) und einer mutmaßlichen guten Vertrautheit von luxemburgischen Schreibern mit französischen Phonem-Graphem-Beziehungen, wäre eine unveränderte Übernahme in Lautung wie Schreibung denkbar. Andererseits kann davon ausgegangen werden, dass den Luxemburgern die französische Lautung stärker präsent war als in anderen germanophonen Gebieten, so dass sich die auditive Rezeption auch in der Schreibung durchgesetzt hat. Dementsprechend wäre zu vermuten, dass vor allem ersteres Szenario der Assimilation, d.h. Beibehaltung der Phonologie - dagegen Änderung der Graphematik, in den Daten zu finden ist.

Tabelle 1 enthält die Ergebnisse für zwei Grapheme mit Akzentzeichen, die nicht zum deutschen Grapheminventar gehören: ${ }^{5}$

Tab. 1: Assimilation der Fremdgrapheme mit Diakritika

\begin{tabular}{|c|c|c|c|c|}
\hline & \multicolumn{2}{|c|}{$\langle\hat{0} / 0: /\rangle \rightarrow\langle 0 / 0: /\rangle$} & \multicolumn{2}{|c|}{$\langle e ́ / e /\rangle \rightarrow\langle e / e /\rangle$} \\
\hline & $\langle\hat{\imath}\rangle$ & $\langle 0\rangle$ & 〈és & $\langle e\rangle$ \\
\hline 1794-1814 & 0 & $146(100 \%)$ & 0 & 1340 (100\%) \\
\hline 1815-1824 & 0 & $12(100 \%)$ & $10(2,4 \%)$ & $402(97,6 \%)$ \\
\hline 1825-1839 & 0 & $3(100 \%)$ & 0 & 477 (100\%) \\
\hline 1840-1849 & 0 & $7(100 \%)$ & 0 & $491(100 \%)$ \\
\hline 1850-1859 & 0 & 0 & 0 & 257 (100 \%) \\
\hline 1860-1869 & 0 & $1(100 \%)$ & 0 & 191 (100 \%) \\
\hline 1870-1879 & 0 & 0 & 0 & 127 (100 \%) \\
\hline 1880-1889 & 0 & $2(100 \%)$ & 0 & 136 (100 \%) \\
\hline 1890-1899 & 0 & 0 & 0 & 200 (100\%) \\
\hline 1900-1909 & 0 & 0 & 0 & 164 (100\%) \\
\hline 1910-1920 & 0 & 0 & 0 & 111 (100 \%) \\
\hline gesamt & 0 & $171(100 \%)$ & $10(0,3 \%)$ & 3896 (99,7 \%) \\
\hline
\end{tabular}

5 Notation basierend auf Heller \& Walz 1992. 
Es lässt sich erkennen, dass es bezüglich Diakritika eine starke Assimilationstendenz gibt, die sich darin äußert, dass sie über den gesamten Untersuchungszeitraum zu (fast) 100\% weggelassen werden. Betroffene Lexeme sind dabei z.B. die Monatsnamen Nivôse und Ventôse des französischen Revolutionskalenders, aber auch z.B. die Nomen Depôt bzw. Réglement und Département. Die Schreibung ohne Akzentzeichen entspricht den heimischen Strukturgesetzen, denen Zirkumflex und Akut fremd sind. Der ursprüngliche Lautwert bleibt dabei bestehen. Da diese Akzentzeichen im Deutschen unbekannt sind, gibt es entsprechend auch keine Phonem-Graphem-Beziehung, auf die bezüglich eines (anderen) Phonems zurückgegriffen und von der die Aussprache abgeleitet werden könnte. Stattdessen werden die Wörter gemäß den Phonem-Graphem-Korrespondenzen für die entsprechenden Grapheme ohne Diakritika ausgesprochen, was auch der französischen Lautung entspricht.

Darüber hinaus könnte die Tatsache, dass es sich um Schriftzeichen aus einem fremden Alphabet handelt, drucktechnische Schwierigkeiten mit sich bringen: „Fremde Zeichen stammen überwiegend aus dem Französischen. Sie werden schon aus schreibtechnischen Gründen häufig getilgt, ähnlich wie die Umlautspunkte deutscher Lehnwörter im Englischen.“ (Munske 1997: 78) Diese Beobachtung wird im vorliegenden Korpus durch die Ausnahmen von der Assimilation von 〈é` gestützt. Alle zehn Belege dafür stammen aus einer einzigen Bekanntmachung (aus dem Intervall 1815-1824) und beziehen sich auf das Lexem Maréchaussée $^{6}$ (eine militärisch organisierte Polizeitruppe), das dort jedes Mal in anderer Schriftart steht als der Rest des (deutschen) Textes (d.h. in Antiqua statt Fraktur). Das könnte darauf hindeuten, dass es im Setzkasten für die deutsche Fraktur keine französischen Akzentzeichen gab und schon alleine deshalb eine graphemische Assimilation erfolgte.

Es existiert außerdem eine aktuelle, vergleichbare Analyse zur Entwicklung der Fremdwortschreibung im Deutschen im 19. Jahrhundert von Anne Zastrow, die als Vergleichsfolie für die luxemburgischen Ergebnisse herangezogen werden kann. Diese erbrachte u.a., dass ,bei den Akzentgraphemen von einer Gesamtassimilationsquote von fast 100\% gesprochen werden“ kann (Zastrow 2015: 363). Die luxemburgischen Daten entsprechen damit allgemein zu beobachtenden Entwicklungen.

Tabelle 2 listet die Ergebnisse für drei weitere Phonographeme auf:

6 Dabei geht es um 〈é/e/〉 im Inlaut und nicht um «ée/e/〉 im Auslaut. Die vorliegende Analyse betrifft nur Grapheme in ersterer Position. 
Tab. 2: Assimilation von Fremdgraphemen mit Entwicklung

\begin{tabular}{|c|c|c|c|c|c|c|}
\hline & \multicolumn{2}{|c|}{ 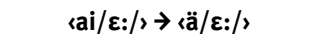 } & \multicolumn{2}{|c|}{$\langle\mathrm{u} / \mathrm{y}: /\rangle \rightarrow\langle\mathrm{ü} / \mathrm{y}: /\rangle$} & \multicolumn{2}{|c|}{$\langle\mathrm{ch} / \mathrm{J} /\rangle \rightarrow\langle\operatorname{sch} / \mathrm{J} /\rangle$} \\
\hline & 〈ai> & 〈ä〉 & $\langle u\rangle$ & $\langle\ddot{u}\rangle$ & 〈ch» & 〈sch $\rangle$ \\
\hline 1794-1814 & $289(25,7 \%)$ & $835(74,3 \%)$ & $172(89,6 \%)$ & $21(10,9 \%)$ & $7(87,5 \%)$ & $1(12,5 \%)$ \\
\hline $1815-1824$ & $185(41,5 \%)$ & $261(58,5 \%)$ & $53(41,4 \%)$ & $75(58,6 \%)$ & $13(35,1 \%)$ & 24 (64,9\%) \\
\hline 1825-1839 & $134(36,3 \%)$ & $235(63,7 \%)$ & $23(21,1 \%)$ & $86(78,9 \%)$ & $15(30,6 \%)$ & $34(69,4 \%)$ \\
\hline 1840-1849 & $57(12,2 \%)$ & $411(87,8 \%)$ & $13(8,1 \%)$ & $147(91,9 \%)$ & $12(22,2 \%)$ & $42(77,8 \%)$ \\
\hline 1850-1859 & $13(7,2 \%)$ & $168(92,8 \%)$ & $4(16,7 \%)$ & $20(83,3 \%)$ & 0 & 15 (100\%) \\
\hline 1860-1869 & $5(4 \%)$ & $121(96 \%)$ & $6(16,2 \%)$ & $31(83,8 \%)$ & $1(16,7 \%)$ & $5(83,3 \%)$ \\
\hline 1870-1879 & $3(3,3 \%)$ & $88(96,7 \%)$ & $2(8,3 \%)$ & $22(91,7 \%)$ & $11(73,3 \%)$ & $4(26,7 \%)$ \\
\hline 1880-1889 & 0 & $74(100 \%)$ & $1(12,5 \%)$ & $7(87,5 \%)$ & $1(50 \%)$ & $1(50 \%)$ \\
\hline 1890-1899 & 0 & $152(100 \%)$ & $2(2,4 \%)$ & $82(97,6 \%)$ & $18(94,7 \%)$ & $1(5,3 \%)$ \\
\hline 1900-1909 & 0 & $132(100 \%)$ & $4(9,8 \%)$ & $37(90,2 \%)$ & $1(50 \%)$ & $1(50 \%)$ \\
\hline 1910-1920 & 0 & $107(100 \%)$ & 0 & $28(100 \%)$ & 0 & $1(100 \%)$ \\
\hline gesamt & $686(21 \%)$ & 2584 (79\%) & $280(33,5 \%)$ & $556(66,5 \%)$ & $79(37,6 \%)$ & $131(62,4 \%)$ \\
\hline
\end{tabular}

Für die graphische Wiedergabe des $/ \varepsilon$ :/ als 〈ai〉 (frz.) oder 〈ä〉(dt.) gibt es anfänglich noch starke Variation, die im zweiten Intervall sogar noch einmal zunimmt und einem Verhältnis von 50:50 nahe kommt. Danach steigt der Anteil der Verschriftlichung nach deutschen Strukturgesetzen kontinuierlich und deutlich an, bis Mitte des Jahrhunderts 90\% und 1880 100\% erreicht sind. Von da an gibt es bis zum Ende des Untersuchungszeitraums keine Veränderung mehr. Lexemgebundene Entwicklungen können hier weniger ausgemacht werden; fast alle Lexeme erscheinen in beiden Varianten. Es handelt sich somit um eine grundsätzlich assimilationsfreudige Variable. Mit der Realisierung der 〈ä〉-Variante geht daneben auch die Tilgung des stummen «e` am Wortende einher - so gibt es z.B. keinen Beleg für die Schreibung «Märe〉,Bürgermeister‘. Hierin deutet sich eine gewisse Implikationsbeziehung zwischen verschiedenen Assimilationsphänomenen an. Die diachrone Entwicklung entspricht in etwa dem, was bei Heller \& Walz (1992), einer weiteren Untersuchung der Fremdwortschreibung im Deutschen, beschrieben wird. Dort wird für das Jahr 1801 (im Wörterbuch von Campe) zu 50\% 〈ä〉-Schreibung festgestellt, ab 1880 dann zu 100\%. Auch Zastrow (2015: 373-374) diagnostiziert für dieses Graphem eine starke Assimilationstendenz: „Die Grapheme $\langle a i / \varepsilon />$ und $\iota \mathrm{ai} / \varepsilon$ :/> zeigen eine - verglichen mit der allgemeinen Assimilationsquote des Jahrhunderts - überdurchschnittliche Quote von 52,10\% und [...] 
[einen] großen Sprung zwischen 1860 und 1890 von 39,57\% auf 78,57\%.“ Wenn bei ihr die Prozentwerte dennoch niedriger ausfallen, liegt das zum einen daran, dass ihre Ergebnisse typebezogen, die der vorliegenden Analyse dagegen tokenbasiert errechnet wurden. Zum anderen liegen der vorliegenden Untersuchung ausschließlich Lexeme mit den Endungen -än oder -är zugrunde, die sich auch in anderen Studien sehr assimilationsfreudig zeigen: „Auch hier lässt sich die Quote mitsamt ihrer Entwicklung besonders auf zwei konkrete Strukturtypen zurück-

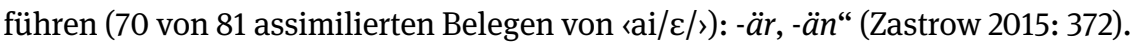

Bei der Schreibung für /y:/ gibt es bis einschließlich des dritten Intervalls beträchtliche Variation. Im ersten Intervall ist die $\langle\ddot{u}\rangle$-Variante sogar noch deutlich unter einem Wert, bei dem man von einer Etablierung sprechen könnte. $\mathrm{Zu}$ diesem Zeitpunkt ist die Übernahme der französischen Lautung schriftlich also kaum erkennbar. Anschließend gibt es eine starke Zunahme der assimilierten Variante, so dass ab 1840 die Prozente zwischen 83\% und 97\% schwanken. Ihre Durchsetzung als Norm ist somit klar erkennbar. Am Ende des Untersuchungszeitraums, d.h. im zweiten Jahrzehnt des 20. Jahrhunderts werden schließlich $100 \%$ assimilierte Realisierung erreicht. Es handelt sich somit um eine Variable mit Entwicklung, die im Schnitt für das gesamte Jahrhundert auf eine Assimilationsquote von $66,5 \%$ kommt. Die stichprobenartige Untersuchung von Heller \& Walz (1992) erbrachte erneut ähnliche Ergebnisse: Anfang des 19. Jahrhunderts (d.h. bei Campe 1801) findet sich noch ausschließlich das Graphem «u〉, in späteren Wörterbüchern dann tendenziell stärker 〈ǜ. Zastrow (2015: 373) beschreibt für ihre Daten ebenfalls eine mittelhohe Assimilationsfreudigkeit, d.h. eine Quote von 47,5\%. ${ }^{7}$

Der Frikativ / $\delta /$ erreicht über den gesamten Untersuchungszeitraum berechnet einen ähnlich hohen Wert hinsichtlich einer assimilierten Schreibung, d.h., der französische Lautwert wurde übernommen und nach deutschen Strukturgesetzen verschriftlicht. Allerdings lässt sich weder eine gleichbleibend hohe Quote noch eine Entwicklung beobachten, vielmehr schwanken die Prozentwerte intervallspezifisch sehr stark. Dies ist auf eine Lexem- bzw. Positionsabhängigkeit der Assimilation zurückzuführen, wobei sich anlautendes «ch / / / > konträr zu der Realisierung im Inlaut verhält. So auch bei Zastrow (2015: 371): „Es ist im Usus festzustellen, dass in 27 von insgesamt 29 nicht assimilierten Wörtern die Anlautposition betroffen ist (z. B. Chaiselongue, Champagner, charmant, Chaussee, Chef, Chevalier, chimärisch). Hier ist das Vorhandensein einer Vielzahl analoger assimi-

7 Damit liegt der Wert erneut unter dem der vorliegenden Studie. Auch hier sollte die unterschiedliche Berechnungsgrundlage berücksichtigt werden. Des Weiteren ist die relative Einordnung zu anderen Variablen bzw. die Bewertung der Autorin zu beachten. 
lierter Fälle (Schaluppe, Scharlatan, Scharnier, Schick) anscheinend nicht Grund genug für eine Assimilation." Entsprechend findet sich auch in den luxemburgischen Daten das Lexem Depesche ausschließlich in der assimilierten Variante Schokolade, Charpie dagegen nur in der «ch〉-Schreibweise. Eine Ausnahme bildet das Nomen Affiche $^{8}$, das in beiden Varianten belegt ist.

Weniger graphisch integriert zeigen sich Fremdwörter mit «ou/u/ s sowie mit «eau/o:/〉, wie Tabelle 3 zeigt:

Tab. 3: Assimilationsresistente Fremdgrapheme

\begin{tabular}{|c|c|c|c|c|}
\hline & \multicolumn{2}{|c|}{$<0 u / u /\rangle \rightarrow\langle u / u /\rangle$} & \multicolumn{2}{|c|}{ «eau/o:/〉 $\rightarrow\langle 0 / 0: /\rangle$} \\
\hline & cous & $\langle u\rangle$ & 〈eaus & 〈0〉 \\
\hline 1794-1814 & $24(92,3 \%)$ & $2(7,7 \%)$ & $39(100 \%)$ & 0 \\
\hline $1815-1824$ & $247(97,6 \%)$ & $6(2,4 \%)$ & $112(100 \%)$ & 0 \\
\hline 1825-1839 & $286(100 \%)$ & 0 & $94(100 \%)$ & 0 \\
\hline 1840-1849 & $152(100 \%)$ & 0 & $153(100 \%)$ & 0 \\
\hline 1850-1859 & $2(100 \%)$ & 0 & $21(100 \%)$ & 0 \\
\hline $1860-1869$ & - & 0 & $36(100 \%)$ & 0 \\
\hline 1870-1879 & $4(100 \%)$ & 0 & $21(100 \%)$ & 0 \\
\hline 1880-1889 & - & 0 & $6(100 \%)$ & 0 \\
\hline 1890-1899 & $5(100 \%)$ & 0 & $68(100 \%)$ & 0 \\
\hline 1900-1909 & $2(100 \%)$ & 0 & $37(100 \%)$ & 0 \\
\hline 1910-1920 & $1(100 \%)$ & 0 & $23(82,1 \%)$ & $5(17,9 \%)$ \\
\hline gesamt & $723(98,9 \%)$ & $8(1,1 \%)$ & $610(99,2 \%)$ & $5(0,8 \%)$ \\
\hline
\end{tabular}

Die Variable $\iota \mathrm{ou} / \mathrm{u} />\rightarrow\langle\mathrm{u} / \mathrm{u} />$ ist durch Lexeme wie z. B. souverän oder Boulevard belegt. Hier gibt es nur zwei Wörter, die sich auch mit der Schreibung « $\mathrm{u}$ 〉 finden (Kurier und Burse), alle anderen werden durchgehend mit ‘ou〉 realisiert. Diese Beobachtungen decken sich erneut mit den Befunden von Zastrow: „Im Ganzen wurden auch in der Kodifikation nur Einzelfallassimilationen konstatiert, die sich v.a. in folgenden Belegen im Usus zeigen: Cuvert (neben häufiger Couvert), Kurier

8 Beim Begriff Affiche handelt es sich um ein Synonym für ,öffentliche Bekanntmachung‘ oder ,Plakat‘ (entlehnt aus dem Französischen). 
(neben häufiger Courier), Kautschuk.“ (Zastrow 2015: 373) Abgesehen von diesen Einzelfällen liegt auch 1890 die Assimilationsquote in dieser Studie bei unter 50\%.

Die (Nicht-)Assimilation von «eau/o:/> schließlich ist in den Bekanntmachungen nur durch das Lexem Büro belegt. Erst im letzten Intervall kommt die assimilierte Schreibung 〈o〉 auf. Diese Assimilationsresistenz wird ebenfalls in den anderen beiden Studien beschrieben: „Diese typisch französischen Grapheme zeigen sich äußerst integrationsresistent.“ (Heller \& Walz 1992: 285) „Die wenigen singulären Regeln für «(e)au/o:/> und «au/っ/> reflektiert der assimilationsresistente Usus“" (Zastrow 2015: 374).

\subsection{Adverbialisierung der Tageszeitangaben}

In der zweiten Variable geht es um die Entwicklung der Tageszeitangaben von der Form als erstarrter Genitiv zu einem Adverb. So gehen den Tageszeitangaben in heutiger Form von Adverbien (z.B. morgens, abends) mehrere Entwicklungsstadien mit anderen strukturellen Beschaffenheiten voraus. Basierend auf der Endung auf -s kann dabei als Ausgangsform der Genitiv Singular des Nomens der jeweiligen Tageszeit angenommen werden, z.B. des Morgens oder des Abends. In seiner adverbialen Bedeutung, d.h. als Benennung der Eigenschaft eines Vorgangs oder eines Ereignisses, d.h. konkret seines Zeitpunkts, ist der Kasus jedoch nicht (mehr) produktiv, sondern erscheint als feste phraseologische Verbindung. Dieser erstarrte Genitiv hat sich dann in der Folge weiter abgeschliffen und der Artikel wurde zunehmend weggelassen. Als letzter Schritt wurde die initiale Majuskel durch eine Minuskel ersetzt, wie es im Gegenwartsdeutschen für Adverbien die Regel ist.

In den Grammatiken und Wörterbüchern lässt sich speziell für die Tageszeitangaben kein Hinweis auf die zeitliche Situierung dieser Entwicklung finden. Bei Elspaß (2005) gibt es jedoch einige Beispiele, die die Variation zwischen der ersten und der zweiten Stufe illustrieren. Diese Beispiele entstammen Auswandererbriefen aus dem 19. Jahrhundert, Beispiel (1) aus dem Jahr 1847.

(1) Den 18ten Aapril sind wier Morgens 10 Ur von Arnien gefaren und des Abens 5 Ur [in] Rotterdam angekommen [...]. (Elspaß 2005: 336, Hervorhebung R.B.)

Der Zeitraum der Adverbialisierung der Tageszeitangaben im Deutschen scheint also deckungsgleich zu sein mit dem Untersuchungszeitraum des Projekts, so dass auch in den vorliegenden Korpusdaten einer entsprechenden Variation nachzugehen lohnt.

Neben der innersprachlichen Motivation der Adverbialisierung ist aber zu vermuten, dass in den öffentlichen Bekanntmachungen der Stadt Luxemburg ein 
Zusammenhang zwischen der Realisierung der Tageszeitangabe im deutschen Text und dem äquivalenten Ausdruck im französischen Paralleltext besteht, d.h., dass verschiedene Formen nicht frei variieren bzw. nicht konsequent zeitlich komplementär distribuiert sind, sondern ihre Wahl vom französischen Äquivalent beeinflusst ist. So lässt sich folgende Kookkurrenz vermuten:
(2) des
Morgen-s
$\leftrightarrow \quad d u$
matin
ART:NEUT.SG.GEN N(,Morgen')-NEUT.SG.GEN
PRART:MASK N(,Morgen'):MASK.SG
(3) Morgens
$\leftrightarrow \quad$ (le) matin
$\operatorname{ADV}(<\mathrm{N}($,Morgen')-NEUT.SG.GEN)
ART:MASK N(,Morgen'):MASK.SG

Die phraseologische Konstruktion mit erstarrtem Genitiv, d.h. die Nominalphrase inklusive Artikel, erscheint parallel zu der Präpositionalphrase du matin im Französischen, in der die Präposition de und der Maskulinartikel le zur Form $d u$ verschmolzen sind. Hier wie auch allgemein wird die Präpositionalphrase de im Französischen dort verwendet, wo im Deutschen der Genitiv steht. Die monolexikalische Variante ist hingegen dort zu vermuten, wo im Französischen keine Präpositionalphrase steht, sondern eine einfache Nominalphrase (mitunter auch ohne Artikel). Abbildung 1 visualisiert zunächst den Prozess der Adverbialisierung für die Tageszeit Morgen.

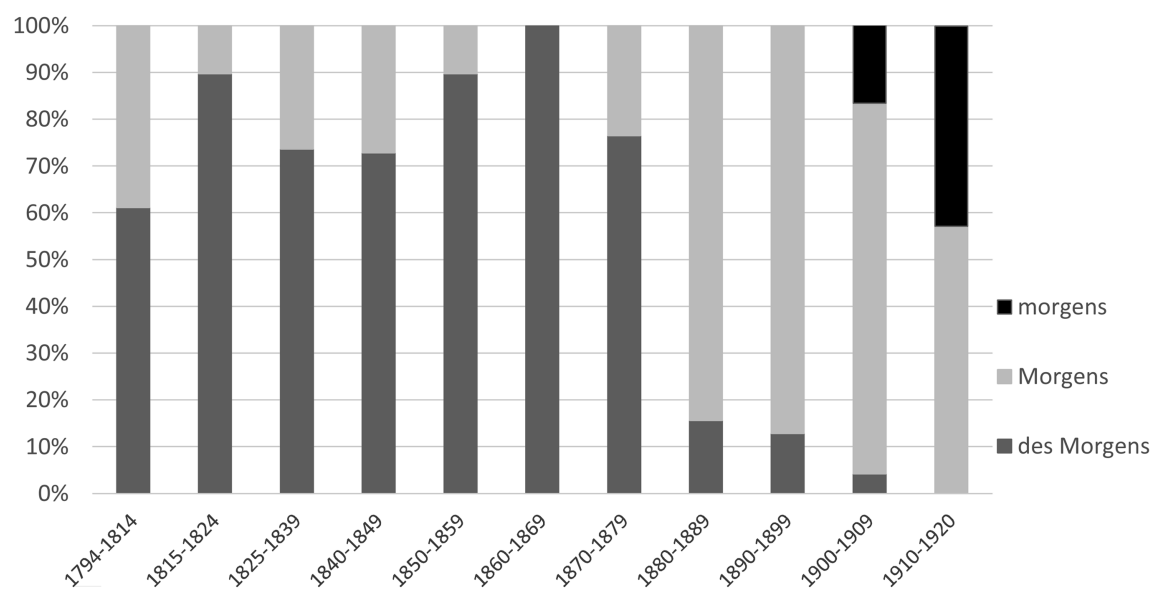

Abb. 1: Adverbialisierung für die Tageszeit Morgen $(\mathrm{N}=431)$

Der oben beschriebene Entwicklungspfad des Morgens $>$ Morgens $>$ morgens ist insofern deutlich erkennbar, als dass die Präferenz der verschiedenen Formen jeweils zeitlich begrenzt wie auch versetzt ist. Für die Adverbialisierung lässt sich somit eine bestimmte Abfolge der Stufen ausmachen. Aber auch Aspekte der 
Standardisierung lassen sich identifizieren. So gibt es am Anfang Variation zwischen der phraseologischen Konstruktion mit erstarrtem Genitiv und der monolexikalischen Form. Erstere ist dabei die präferierte Variante, deren Anteil bis zum Intervall 1860-69 bis auf 100\% ansteigt (wenn auch nicht kontinuierlich). Die erste Hälfte des 19. Jahrhunderts ist also durch Variantenreduktion gekennzeichnet. Anschließend nimmt die Verwendung der monolexikalischen Variante (mit Majuskel) wieder stark zu und es kommt zu einem Variantenwechsel. Ab 1900 setzt die letzte Stufe der Adverbialisierung ein und Formen mit wortinitialer Minuskel kommen vermehrt auf. Dieser Trend dürfte sich nach dem Untersuchunsgszeitraum fortgesetzt haben, ist aber nicht mehr durch die Projektdaten belegt.

In einem nächsten Analyseschritt gilt es, die Verbindung zum französischen Paralleltext zu ziehen und zu berücksichtigen, welche Konstruktionen dort äquivalent verwendet werden. Abbildung 2 schlüsselt auf, welche französischen Formen den verschiedenen Belegen im Deutschen gegenüberstehen. Dabei symbolisieren die drei verschiedenen Graustufen - wie in Abbildung 1 - die drei Stufen der Adverbialisierung im Deutschen (des Morgens $=$ dunkelgrau, Morgens $=$ hellgrau, morgens = schwarz). Die verschiedenen Schraffuren sind stellvertretend für die Varianten im Französischen, d.h., eine einfarbige Füllung der Säulen verweist auf die Verwendung der Präpositionalphrase, schräge Schraffur auf ein Nomen ohne Artikel usw. (vgl. die Legende). Es gibt schließlich auch Fälle, in denen die Tageszeitangabe im Deutschen keine Entsprechung im französischen Paralleltext findet; diese werden mit „keine Übersetzung“ bezeichnet. Sollte sich die Hypothese bestätigen, wären die dunkelgrauen Anteile (= Nominalphrase mit Artikel im Deutschen) ausschließlich bzw. überwiegend einfarbig, d.h. hätten du matin als französisches Äquivalent. Die hellgrauen Anteile (= einfache Nominalphrase im Deutschen) hingegen wären entsprechend ausschließlich bzw. überwiegend (schräg) schraffiert (das parallele Vorkommen von (le) matin im Französichen symbolisierend). 


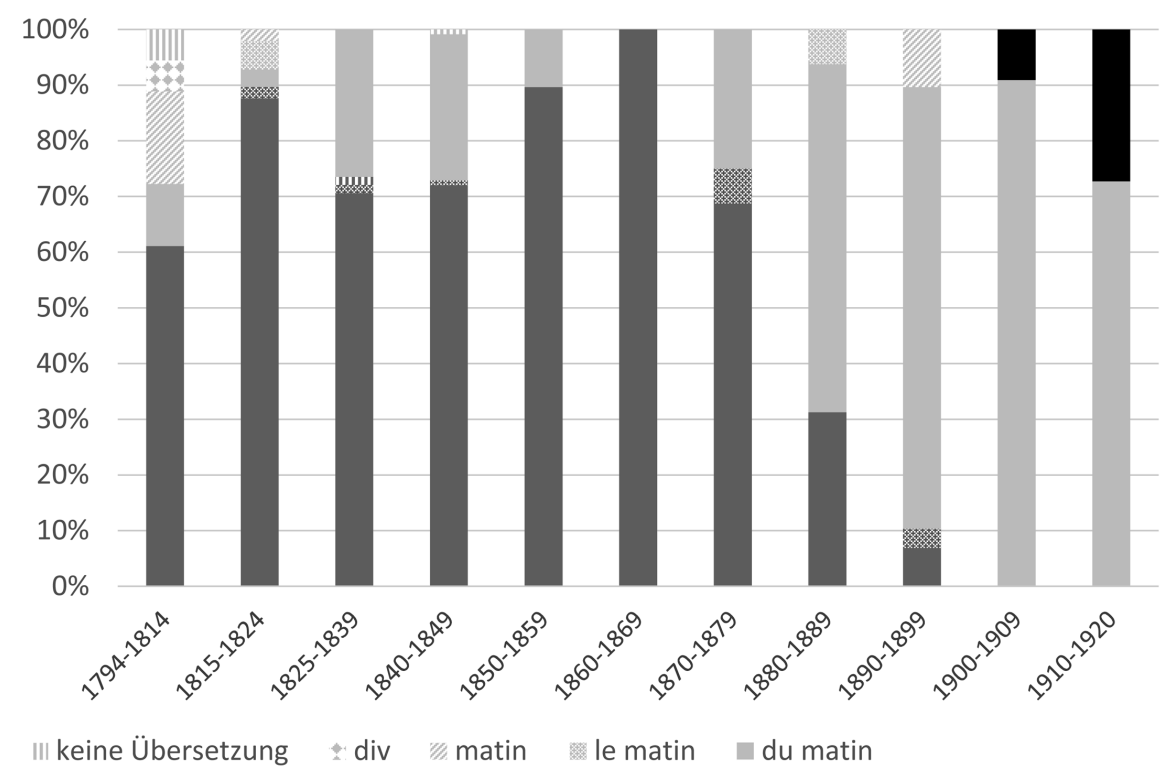

Abb. 2: Adverbialisierung für die Tageszeit Morgen unter Berücksichtigung des frz. Äquivalents

Das Diagramm lässt sich folgendermaßen interpretieren: Die phraseologische Verbindung mit erstarrtem Genitiv des Morgens (dunkelgraue Säulen) zeigt auffällig hohe Kookurrenz mit frz. du matin (einfarbige Füllung), während die monolexikalische Form Morgens (ohne Genitiv-Artikel) parallel zu matin oder le matin steht. Diese Zuordnung lässt sich indes nur für die ersten Dekaden des 19. Jahrhunderts beobachten. Ab ungefähr 1825 steigt die Zahl der adverbialisierten Form im Kontext von du matin, womit die vorherige Kookkurrenzregel gewissermaßen überschrieben wird. Die monolexikalische Variante nimmt anschließend jedoch wieder ab. Von 1860-69 entfallen 100\% der Belege auf die Form des Morgens, so dass sich keine (systematische) Kovariation erkennen lässt. Im Französischen wurde (zwar) analog ausschließlich du matin realisiert, ein Zusammenhang kann jedoch insofern ausgeschlossen werden, als die deutsche Realisierung zuvor schon aus der Abhängigkeit vom französischen Paralleltext getreten ist. Vielmehr kann hier (für das Deutsche) von einer Standardisierung ausgegangen werden. Danach nimmt der Anteil der phraseologischen Form jedoch in großen Schritten ab und die monolexikalische Form ist unabhängig von der französischen Entsprechung die deutlich präferierte Variante. Auch der letzte Schritt der Adverbialisierung geschieht unabhängig vom Französischen: Im Kontext derselben französischen Konstruktion wechselt im Deutschen schließlich die wortinitiale Schreibung von Majuskel auf Minuskel. 
Diese Analyse wurde auch für weitere Tageszeitangaben durchgeführt. Abbildung 3 stellt die Entwicklungen für die Tageszeit Abend dar. Sie enthält bereits die Differenzierung nach den französischen Äquivalenten. Das Diagramm ist nach demselben Schema aufgebaut wie jenes für die Tageszeitangabe Morgen, d.h., die drei verschiedenen Graustufen bilden die drei Stufen der Adverbialisierung im Deutschen ab (des Abends $=$ dunkelgrau, Abends = hellgrau, abends $=$ schwarz) und die unterschiedlichen Schraffuren verschiedene Formen im französischen Paralleltext. In der Legende sind die belegten Kookkurrenzen samt entsprechender Füllung der Säulen einzeln aufgelistet.

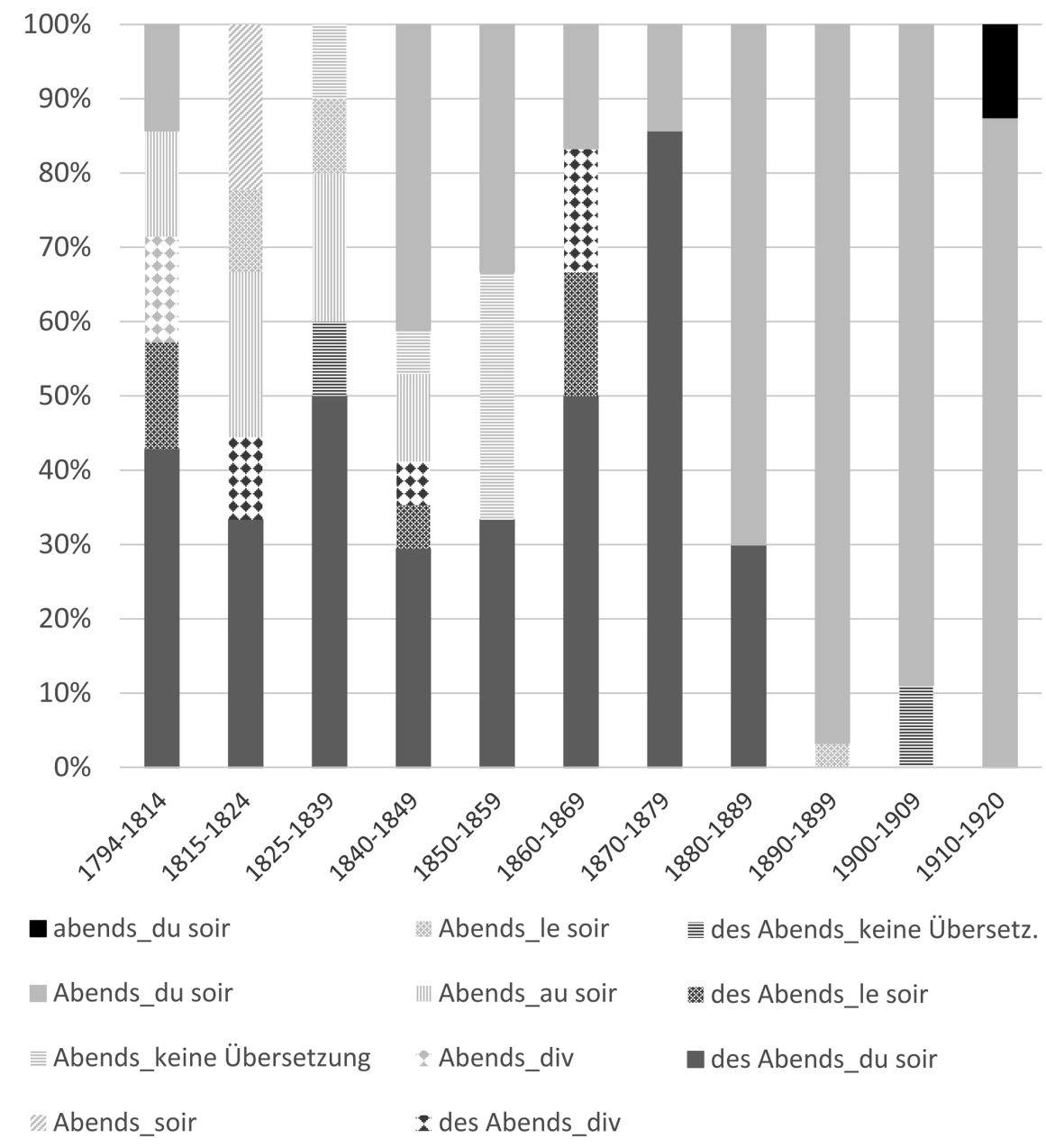

Abb. 3: Adverbialisierung für die Tageszeit Abend $(\mathrm{N}=116)$ 
Es sind ähnliche Entwicklungen wie für die Tageszeitangabe Morgen zu beobachten. Auch hier gibt es eine Periode der Variation zwischen der phraseologischen Verbindung mit erstarrtem Genitiv und der abgeschliffenen monolexikalischen Variante. Darauf folgt eine Variantenreduktion zugunsten der polylexikalischen Konstruktion, der dann wiederum zeitlich geordnete Variantenwechsel von des Abends auf Abends und (vermutlich) weiter auf abends folgen, die als Adverbialisierung eingeordnet werden können. Auch in Bezug auf die französische Parallelform lassen sich ähnliche Beobachtungen machen. So erscheint des Abends (dunkelgraue Säulen) zusammen mit frz. du soir (einfarbige Füllung), während die monolexikalische Form Abends (ohne Genitiv-Artikel, hellgraue Säulen) meistens parallel zu anderen Konstruktionen steht. Diese Kookkurrenzregel wird im Laufe des Jahrhunderts aufgehoben und die deutsche Realisierung erfolgt unabhängig vom französischen Paralleltext, so dass im Kontext ein und derselben französischen Form im Deutschen eine Veränderung der Formen zu konstatieren ist. Allerdings sind diese Entwicklungsschritte im Vergleich zu Morgens zeitlich versetzt und finden in etwa eine Dekade später statt. So erreicht die phraseologische Verbindung mit erstarrtem Genitiv für die Tageszeit Morgen im Intervall 1860-69 ihren höchsten Prozentwert, für die Tageszeit Abend hingegen 1870-79. Die Überschreibung des Kookkurrenzmusters ist außerdem für die Tageszeitangabe Morgen bei 1825-39 angesiedelt, für den Abend lässt sie sich erst für 1840-49 erkennen. Daneben sind die prozentualen Verhältnisse in der ersten Hälfte des 19. Jahrhunderts, d.h. vor der temporären Durchsetzung der polylexikalischen Variante, durchaus unterschiedlich. Während für Morgen die polylexikalische Variante in der gesamten ersten Hälfte des 19. Jahrhunderts die präferierte Variante darstellt, ist die Variation für Abend sehr viel größer. Hier gibt es auch Intervalle (1815-1824, 1840-1849, 1850-1859), in denen die monolexikalische Form den überwiegenden Teil der Belege ausmacht. Dies scheint zum Teil durch die Realisierung des französischen Äquivalents (1815-1824: ca. 67\% au soir, soir u.a.) und die beschriebene Abhängigkeit der deutschen Form bedingt zu sein, gilt aber auch grundsätzlich. ${ }^{9}$

9 Vgl. z.B. das Intervall 1840-1849, in dem im Kontext von frz. du soir mehrheitlich Abends verwendet wird. 


\subsection{Genusdifferenzierung beim Zahlwort ,zwei}

In der dritten Variablen geht es um die vom Genus des Bezugsnomens abhängige formale Gestalt des attributiv verwendeten Zahlworts, für die es folgende Beobachtung gibt:

Das Zahlwort zwei kannte im Mhd. genusdifferenzierte Formen, die aber bis Ende des 18. Jahrhunderts nach dem ursprünglichen Neutrum zwei ausgeglichen wurden. Danach kommen genusdifferenzierte Formen nur noch mundartlich vor. (Durrel \& Whitt 2013: 108)

Wenn die öffentlichen Bekanntmachungen aus Luxemburg auf unterschiedliche Formen des Zahlworts hin untersucht werden, geht es dementsprechend in erster Linie um die Nachverfolgung einer gesamtdeutschen Entwicklung, d.h. der Zurückdrängung der Genusdifferenzierung aus der deutschen Schriftsprache. Jedoch wird hier auch die dialektgeographische Situation von Luxemburg relevant, denn:

[h]insichtlich der Formenbildung der Zahlwörter ist in den hochdeutschen Dialekten im Westen und Süden auf die weitverbreitete Bewahrung des alten Unterschiedes zwischen den drei Geschlechter von ,zwei‘ hinzuweisen (ahd. Mask. zwēne, Fem. zwā, zwō, Neutr. zwei), z.B. [...] südfrk. Rap. tswē, tswū, tswai; [...]. (Schirmunski 1962: 474).

Folglich gehört das Westmoselfränkische zu den Dialekten, in denen die Unterscheidung beibehalten wurde, genauso wie das daraus emergierende Luxemburgische, wie die entsprechenden Einträge im Wörterbuch der luxemburgischen Mundart von 1906 belegen. So wird für zwê angegeben: „zwei vor einem sächlichen Substantiv; zwê Kanner“. Bezüglich $z w e^{i} n$ heißt es dort: „Zwei (vor einem männlichen Substantivum); zwei ${ }^{10}$ Männer“ und für $z w o^{u}$ schließlich: „zwei (vor weiblichen Substantiven); zwo ${ }^{u}$ Frâen“. Es ist somit zu vermuten, dass die lokale Varietät eine Fortführung der Genusdifferenzierung im Schriftdeutschen stützt. Diese Hypothese wird auch durch die Beobachtung von Durrell \& Whitt (2013) unterlegt, die schon in Zeitungsdaten des 17. Jahrhunderts genusdifferenzierte Formen nur noch dort fanden, „wo diese Varianten im Dialekt noch vorkommen“ (Durrell \& Whitt 2013: 108-109). Dabei heben sie besonders das Westmitteldeutsche hervor, zu dem eben auch das (West-)Moselfränkische gehört. Bei dieser Variablen überschneiden sich somit diatopische und historische Motivationen für eine Variation - in jedem Fall aber geht es um sprachinterne Entwicklungen.

10 Im vorliegenden Beispiel wurde das wortfinale - $n$ gemäß der sog. ,Eifler Regel` getilgt (vgl. Gilles 2006). 
Tabelle 4 fasst die Ergebnisse für die luxemburgischen Bekanntmachungen des 19. Jahrhunderts intervallweise zusammen:

Tab. 4: Formen des Zahlworts ,zwei‘

\begin{tabular}{l|r|r|r|r}
\hline & \multicolumn{2}{|c|}{ zween } & zwo & zwei \\
\hline $1794-1814$ & Maskulin & Neutrum & Fem & Mask. Neutr. Fem \\
\hline $1815-1824$ & 10 & 2 & 7 & $125(86,8 \%)$ \\
\hline $1825-1839$ & 4 & 0 & 8 & $83(87,4 \%)$ \\
\hline $1840-1849$ & 2 & 0 & 4 & $117(95,1 \%)$ \\
\hline $1850-1859$ & 0 & 0 & 0 & $81(100 \%)$ \\
\hline $1860-1869$ & 0 & 0 & 0 & $26(100 \%)$ \\
\hline $1870-1879$ & 0 & 0 & 0 & $24(100 \%)$ \\
\hline $1880-1889$ & 0 & 0 & 0 & $16(100 \%)$ \\
\hline $1890-1899$ & 0 & 0 & 0 & $22(100 \%)$ \\
\hline $1900-1909$ & 0 & 0 & 0 & $16(100 \%)$ \\
\hline $1910-1920$ & 0 & 0 & 0 & $6(100 \%)$ \\
\hline
\end{tabular}

Der Tabelle lässt sich entnehmen, dass es im Korpus unterschiedliche Formen für das Zahlwort ,zwei' gibt: Neben zwei sind auch zween und zwo belegt. Diese Variation ist allerdings zeitlich begrenzt: $\mathrm{Ab}$ dem dritten Intervall liegt die Gebrauchshäufigkeit der Einheitsform zwei bei $95 \%$ oder höher, womit ihre Durchsetzung als Norm konstatiert werden kann. Ihr Anteil liegt bereits im ersten Intervall bei über $86 \%$ - ein Hinweis darauf, dass der Ausgleichsprozess - wie in der Forschung beschrieben - schon früher eingesetzt hat. Auch eine nach dem Genus des Bezugsnomens unterschiedliche Realisierung ist zu erkennen. So taucht $z w o$ ausschließlich mit dem Femininum auf, zween dagegen steht überwiegend mit Nomen im Maskulinum. Gleichzeitig ist das Aufbrechen des Systems auch qualitativ evident, da z.B. zwo zwar ausschließlich mit Nomen im Femininum auftritt, nicht jedoch umgekehrt alle Feminina ausschließlich mit der Form $z w o$. Auch innerhalb eines Dokuments wird die Genusdifferenzierung nicht konsequent eingehalten. Dementsprechend steht im Plakat mit der Signatur Imp. III_1379 (1820) sowohl „zwo niederländische Ellen“ als auch „zwei Palmen“, d.h. zwei Femininnomina mit unterschiedlicher Form des attributiv verwendeten Zahlworts ,zwei`. Ähnlich gibt es in einem anderen Dokument (LU Imp. II_0286, 1796) 
zwei Maskulinnomina, die mit zwei quantifiziert werden, jedoch einmal mit der Form zween und einmal mit der Form zwey realisiert. Schließlich lässt sich ein Beispiel anführen, in dem nur ein einziges Mal eine von zwei abweichende Form verwendet wird. So finden sich in LU Imp. II_0361 (1796) folgende mit zwei quantifizierte Nomina: „zween Blätter“, „,zwey obigen Beschlüsse“, „zwey ähnliche Duplicata“ sowie „zwey Sprachen“.

An dieser Aufteilung lässt sich des Weiteren erkennen, dass zwar nicht die konkreten (bzw. oberflächlichen) phonologischen Formen aus dem Westmoselfränkischen bzw. Luxemburgischen entlehnt wurden - dort lautet z. B. die Femininform schließlich $z w o^{u}$-, jedoch die Zuordnung Genus - Form übereinstimmt. Unter Berücksichtigung der stattgefundenen Vokalentwicklungen im Luxemburgischen, im Laufe derer ahd. /e/ zu /ei/ bzw. /ô/ zu /ou/ diphthongiert und ahd. /ei/ zu /e/ monophthongiert wurden (Gilles 1999), lässt sich erkennen, dass sich westmoselfränkisches bzw. luxemburgisches und althochdeutsches System (vgl. die Beschreibung bei Schirmunski 1962) entsprechen. Wenn also in den Daten Maskulinnomina mit zween quantifiziert werden, deckt sich dies mit der Realisierung von zwéin vor Nomen im Maskulinum im Luxemburgischen. Schon das Vorkommen der Genusdifferenzierung grundsätzlich könnte durchaus durch die Stabilität dieses Merkmals in der lokalen Varietät gestützt sein, denn es finden sich unterschiedliche Formen in den Daten, obwohl der Untersuchungszeitraum sich knapp nach der in der Literatur angegebenen Periode des Ausgleichs befindet. Die systematische Übereinstimmung spricht umso mehr für solch einen Effekt.

\section{Zusammenfassung}

Unter dem Eindruck der Beobachtung Zollnas (2004: 3192), dass sich frankophoner und germanophoner Sprachraum ,in Auseinandersetzung miteinander entwickelt haben“ und der Feststellung, dass es bezüglich der Entwicklung des Deutschen und seiner Grammatik in Luxemburg unter systematischer Einbeziehung des Sprachkontaktes v.a. mit dem Französischen bislang eine Forschungslücke gibt, wurde für den vorliegenden Beitrag das Ziel gesetzt, den Wandel des Deutschen in Luxemburg im 19. Jahrhundert zu beleuchten und insbesondere Aspekte des Sprachkontakts mit dem Französischen in diesem Zusammenhang zu berücksichtigen. Die Hypothesen bezüglich des Wirkungsgrades bzw. -bereichs waren für die einzelnen Variablen jeweils unterschiedlich, die grundsätzliche Annahme war jedoch stets die, dass sich die Mehrsprachigkeit in der Realisierung der Variablen bemerkbar machen würde. Magenau (1964) beschreibt passend dazu für die erste Hälfte des 20. Jahrhunderts, dass in der Zeitungssprache 
Luxemburgs im Vergleich mit der binnendeutschen mehr fremde Wörter auftauchen, wobei dies Wörter umfasst, die unmittelbar aus dem Französischen kommen als auch Wörter lateinisch-französischer Herkunft, die unter französischem Einfluss häufig verwendet werden. Wenn auch grundsätzlich das „deutsche Sprachgefühl [als] verhältnismäßig sicher“ (Magenau 1964: 100) eingestuft wird, so konstatiert sie aber dennoch: „Der Einfluß der Mundart ist deutlich zu erkennen.“ (Magenau 1964: 102) Im Gesamtbild ergibt sich somit für das Deutsche in Luxemburg bedingt durch den Sprachkontakt ein eigener Charakter.

Die Ergebnisse der hier vorliegenden Analyse weisen jedoch in eine andere Richtung. Die Assimilation von Fremdwörtern aus dem Französischen verläuft je nach Graphem unterschiedlich, so dass die Idee einer eindeutigen Wirkungsweise des Sprachkontakts verworfen werden muss. Vielmehr spielt u.a. die Drucktechnik eine Rolle, aufgrund derer in der deutschen Fraktur keine französischen Akzentzeichen dargestellt werden. Zudem entsprechen die Befunde in vielen Fällen denen, die in vergleichbaren Studien für das deutschländische Sprachgebiet dokumentiert wurden. Wenn dies auch nicht für die konkreten Zahlen zutrifft, dann doch für die grundsätzliche Tendenz der Realisierungen.

Bei der Adverbialisierung der Tageszeitangaben wurde von vorneherein (nur) ein indirekter Einfluss des Französischen zur Diskussion gestellt. Das heißt, es wurde nicht erwartet, dass im Deutschen eine neue Form gebildet wird, die es ohne Sprachkontakt nicht gäbe; vielmehr war die Hypothese, dass eine konkrete Variante aus einem bereits bestehenden Inventar von Varianten gewählt wird. Diese Wahl wird jedoch nach dem Modell der Gebrauchsmuster der Kontaktsprache Französisch getroffen. Aber auch diese Beobachtung trifft nur für die ersten Jahrzehnte des Untersuchungszeitraums zu. Danach entwickelte sich die Struktur unabhängig vom Französischen und der Einfluss der deutschen Standardisierung dominierte.

Die Genusdifferenzierung beim Zahlwort ,zwei` diente gewissermaßen als Kontrollvariable, bei der nicht das Französische, dafür aber der Varietätenkontakt relevant wurde, in dem Sinne, dass er die Fortführung eines alten Merkmals in der Schriftsprache unterstützt. Tatsächlich finden sich auch noch genusdifferenzierte Formen in den Daten, obwohl der Literatur zufolge der Ausgleich im deutschländischen Raum zu dem Zeitpunkt schon abgeschlossen ist. Das Aufbrechen des Systems ist unterdessen klar erkennbar und nach wenigen Dekaden ist die genusneutrale Variante die Norm.

Die systemische Analyse führt somit zu der Erkenntnis, dass die Standardisierung auf sprachsystematischer Ebene ihre volle Wirkung entfaltet und die Mehrsprachigkeit in Luxemburg im Laufe des Jahrhunderts überlagert. 


\section{Literatur}

Baum, Richard (2000), „Französisch als dominante Sprache Europas“, in: Werner Besch / Anne Betten / Oskar Reichmann \& Stefan Sonderegger (Hrsg.), Sprachgeschichte. Ein Handbuch zur Geschichte der deutschen Sprache und ihrer Entstehung, 2. Teilbd., (Handbücher zur Sprach- und Kommunikationswissenschaft, 2.2), 2., vollständig neu bearb. u. erw. Aufl. Berlin / New York, 1107-1117.

Beyer, Rahel (2015), „IDaSTo - Ein Tool zum Taggen und Suchen in historischen Paralleltexten“, in: Bernhard Fisseni / Bernhard Schröder / Torsten Zesch (Hrsg.), GSCL 2015 International Conference of the German Society for Computational Linguistics and Language Technology. Proceedings of the Conference, 162-169 [http://www.gscl.org/proceedings/index.html (letzter Zugriff: 19. 04. 2016)].

Beyer, Rahel / Peter Gilles / Olivier Moliner \& Evelyn Ziegler (2014), „Sprachstandardisierung unter Mehrsprachigkeitsbedingungen: Das Deutsche in Luxemburg im 19. Jahrhundert“, in: Vilmos Ágel \& Andreas Gardt (Hrsg.), Paradigmen der aktuellen Sprachgeschichtsforschung, (Jahrbuch für germanistische Sprachgeschichte, 5), Berlin / Boston, 283-298.

Besch, Werner (2003), „Die Entstehung und Ausformung der neuhochdeutschen Schriftsprache/Standardsprache“, in: Werner Besch / Anne Betten / Oskar Reichmann \& Stefan Sonderegger (Hrsg.), Sprachgeschichte. Ein Handbuch zur Geschichte der deutschen Sprache und ihrer Entstehung, 3. Teilbd., (Handbücher zur Sprach- und Kommunikationswissenschaft, 2.3), 2., vollständig neu bearb. u. erw. Aufl. Berlin / New York, 22522287.

Claridge, Claudia (2008), „Historical Corpora“, in: Anke Lüdeling / Merja Kytö (Hrsg.), Corpus Linguistics. An International Handbook, Teilbd. 1, (Handbücher zur Sprach- und Kommunikationswissenschaft, 29.1), Berlin, 242-259.

Durrell, Martin / Richard J. Whitt (2013), „Zum Abbau der regionalen Variation im Standardisierungsprozess 1650-1800: Belege aus dem GerManC-Korpus“, in: Franciszek Grucza (Hrsg.), Akten des XII. Internationalen Germanistenkongresses Warschau 2010. Vielfalt und Einheit der Germanistik weltweit, Bd. 17, Frankfurt a. M. et al., 107-111.

Elspaß, Stephan (2005), Sprachgeschichte von unten. Untersuchungen zum geschriebenen Alltagsdeutsch im 19. Jahrhundert, (Germanistische Linguistik, 263), Tübingen.

Fehlen, Fernand (2009), BaleineBis: Une enquête sur un marché linguistique multilingue en profonde mutation. Luxemburgs Sprachenmarkt im Wandel, (Recherche, Etude, Documentation, 12), Luxemburg: SESOPI Centre Intercommunautaire.

Fröhlich, Harald (1989), „Dreisprachiges Luxemburg-Sprachkontakt ohne Sprachkonflikt?“, in: Germanistische Mitteilungen, 30, 105-114.

Fröhlich, Harald \& Fernand Hoffmann (1997), „Luxemburg“, in: Hans Goebl / Peter H. Nelde / Zdenek Starý \& Wolfgang Wölck (Hrsg.), Kontaktlinguistik. Ein internationales Handbuch zeitgenössischer Forschung, Teilbd. 2, (Handbücher zur Sprach- und Kommunikationswissenschaft, 12.2) Berlin / New York, 1158-1172.

Gilles, Peter (2006), „Phonologie der n-Tilgung im Moselfränkischen (,Eifler Regel'). Ein Beitrag zur dialektologischen Prosodieforschung“, in: Claudine Moulin \& Damaris Nübling (Hrsg.), Perspektiven einer linguistischen Luxemburgistik. Studien zu Diachronie und Synchronie, (Germanistische Bibliothek, 25), Heidelberg, 29-68.

Gilles, Peter \& Evelyn Ziegler (2013), „The Bilingual Historical Luxembourgish Public Notices Database“, in: Paul Bennett / Martin Durell / Silke Scheible \& Richard J. Whitt (Hrsg.), New 
Methods in Historical Corpus Linguistics, (Corpus Linguistics and Interdisciplinary Perspectives on Language - CLIP, 3), Tübingen, 127-138.

Heine, Bernd (2009), „Identifying instances of contact-induced grammatical replication“, in: Samuel Gyasi Obeng (Hrsg.), Topics in Descriptive and African Linguistics: Essays in Honor of Distinguished Professor Paul Newman, München, 29-56.

Heine, Bernd \& Tania Kuteva (2005), Language Contact and Grammatical Change, (Cambridge Approaches to Language Contact, 3), Cambridge.

Heller, Klaus \& Brigitte Walz (1992), „Zur Geschichte der Fremdwortschreibung im Deutschen. Beobachtungen von Campe bis Duden“, in: Dieter Nerius \& Jürgen Scharnhorst (Hrsg.), Studien zur Geschichte der deutschen Orthographie, (Germanistische Linguistik, 108-109), Hildesheim, 277-338.

Horner, Kristin \& Jean-Jaques Weber (2008), „The Language Situation in Luxembourg“, in: Current Issues in Language Planning, 9, 69-128.

Magenau, Doris (1964), Die Besonderheiten der deutschen Schriftsprache in Luxemburg und in den deutschsprachigen Teilen Belgiens, (Duden-Beiträge, Sonderreihe: Die Besonderheiten der deutschen Schriftsprache im Ausland, 15), Mannheim.

Mattheier, Klaus J. (1998), „Allgemeine Aspekte einer Theorie des Sprachwandels“, in: Werner Besch / Anne Betten / Oskar Reichmann \& Stefan Sonderegger (Hrsg.), Sprachgeschichte. Ein Handbuch zur Geschichte der deutschen Sprache und ihrer Entstehung, Teilbd. 1, (Handbücher zur Sprach- und Kommunikationswissenschaft, 2.1), 2., vollständig neu bearb. u. erw. Aufl. Berlin / New York, 824-836.

Milroy, James (1992), Linguistic Variation and Change. On the historical sociolinguistics of English, (Language in society, 19), Oxford.

Munske, Horst Haider (1997), Orthographie als Sprachkultur. Frankfurt a. M. et al.

Pauly, Michel (2011), Geschichte Luxemburgs, (Beck'sche Reihe, 2732), München.

Rash, Felicity J. (2002), Die deutsche Sprache in der Schweiz: Mehrsprachigkeit, Diglossie und Veränderung. Bern.

Ravida, Fausto (2012), Graphematisch-phonologische Analyse der Luxemburger Rechnungsbücher (1388-1500). Ein Beitrag zur Historischen Stadtsprachenforschung, (Germanistische Bibliothek, 43), Heidelberg.

Schirmunski, Victor (1962), Deutsche Mundartkunde. Vergleichende Laut- und Formenlehre der deutschen Mundarten, (Veröffentlichungen des Instituts für deutsche Sprache und Literatur, 25), Berlin.

Sieburg, Heinz (2013), „Die Stellung der deutschen Sprache in Luxemburg. Geschichte und Gegenwart“, in: Heinz Sieburg (Hrsg.), Vielfalt der Sprachen - Varianz der Perspektiven. Zur Geschichte und Gegenwart der Luxemburger Mehrsprachigkeit, Bielefeld, 81-106.

Sieburg, Heinz (2012), „Luxemburger Deutsch? Zur Frage einer nationalen Varietät der deutschen Standardsprache in Luxemburg“, in: Franciszek Grucza et al. (Hrsg.), Akten des XII. Internationalen Germanistenkongresses Warschau 2010. Vielfalt und Einheit der Germanistik weltweit, Bd. 3, (Publikationen der Internationalen Vereinigung für Germanistik (IVG), 3 ), Frankfurt a. M. et al., 139-143.

Solms, Hans-Joachim / Klaus-Peter Wegera (1999), Zur Luxemburger Druckersprache im 17. Jahrhundert, Luxemburg.

Wörterbuch der luxemburgischen Mundart (1906), Luxemburg.

Zastrow, Anne (2015), Die Entwicklung der Fremdwortschreibung im 19. Jahrhundert. Kodifikation und Usus, (Lingua Historica Germanica, 11), Berlin / Boston. 
Zollna, Isabel (2004), „Französisch und Provencalisch/Deutsch“, in: Werner Besch / Anne Betten / Oskar Reichmann \& Stefan Sonderegger (Hrsg.), Sprachgeschichte. Ein Handbuch zur Geschichte der deutschen Sprache und ihrer Entstehung, Teilbd. 3, (Handbücher zur Sprach- und Kommunikationswissenschaft, 2.3), 2., vollständig neu bearb. u. erw. Aufl. Berlin / New York, 3192-3202. 